\title{
Analysis of microRNAs -15b, -16, -21, -221 and - 222 as molecular markers in the blood of rats submitted to focal cerebral ischemia associated with alcoholism
}

\author{
P.C. Novais ${ }^{1,2}$, F.S.Lizarte Neto ${ }^{1}$, M.L. de A. Cirino ${ }^{1}$, L.B. Porsani ${ }^{1}$, \\ J.P. da Silva ${ }^{1}$, I.S. Gula ${ }^{1}$, M. de T. Durand ${ }^{3}$, C.A.M. de Carvalho ${ }^{1}$, \\ B. C. Schimming ${ }^{4}$, M. de F.G.S. Tazima ${ }^{1}$, C.G. Carlotti $\mathbf{J r}^{1}$, B.O. Colli ${ }^{1}$, \\ D.P. da C. Tirapelli ${ }^{1}$ and L.F. Tirapelli ${ }^{1}$ \\ ${ }^{1}$ Departamento de Cirurgia e Anatomia, Faculdade de Medicina de Ribeirão \\ Preto, Universidade de São Paulo, Ribeirão Preto, SP, Brasil \\ ${ }^{2}$ Faculdade de Odontologia, Universidade de Marília, Marília, SP, Brasil \\ ${ }^{3}$ Faculdade de Medicina, Universidade de Ribeirão Preto, Ribeirão Preto, \\ SP, Brasil \\ ${ }^{4}$ Instituto de Biociências, Universidade Estadual Paulista, Botucatu, Brasil \\ Corresponding author: D.P. da C. Tirapelli \\ E-mail: lab.biomol.cirurgia@fmrp.usp.br
}

Genet. Mol. Res. 19 (3): gmr18637

Received June 09, 2020

Accepted August 11, 2020

Published August 31, 2020

DOI http://dx.doi.org/10.4238/gmr18637

\begin{abstract}
One of the main global causes of mortality is cerebral ischemia. Studies report that alcohol abuse causes health problems, aggravating the conditions of neurological diseases. The role of microRNAs has been highlighted in the literature as biomarkers in various types of diseases, including neurological disorders. Evidence of dysregulation of serum miRNAs related to apoptosis in experimental brain ischemia associated with alcoholism is scarce. We evaluated the gene expression of miRNA-15b and 16 (apoptotic) and miRNA-21, -221 and -222 (anti-apoptotic) in the blood after experimental induction of temporary focal ischemia (90 minutes) by occlusion of the middle cerebral artery in rats, followed by reperfusion for 48 hours, associated with a chronic alcoholism model. Fifty-six rats were randomly divided into seven experimental groups: Control (C); Sham (S); Ischemic (I); Ischemia-reperfusion
\end{abstract}


(IR), Alcoholic (A); Alcoholic and ischemic (I + A); Ischemiareperfusion and Alcoholic (IR $+\mathrm{A})$. The blood samples were collected for gene expression analysis of some serum miRNAs by PCR in real time. The serum expression of miRNA-16 was higher in the IR group compared to $\mathrm{C}$ and $\mathrm{S}$ groups $(\mathrm{P}<0.05)$ but no association with chronic alcoholism was found. The serum expressions of miRNA-21, -221 and -222 were low in all groups and were not correlated with ischemic injury and/or chronic alcoholism. The serum expressions of miRNA-15b, $-21,-221$ and -222 were similar among the experimental groups, with no correlation with ischemia, with or without reperfusion, and/or alcoholism. The overexpression of miRNA-16 in the blood of I and IR groups demonstrated a correlation with the ischemic process, mainly after reperfusion for 48 hours, associated or not with alcoholism.

Key words: Cerebral Ischemia; Apoptosis; Alcoholism; Morphology; miRNA; Blood

\section{INTRODUCTION}

Annually, about 15 million people suffer stroke. It is still described that approximately 5 million lose their lives while another 5 million have some type of disability. This interruption in the tissue blood supply of the brain can be caused by ischemic or hemorrhagic origin. Ischemic stroke is responsible for $87 \%$ of all clinically presented subtypes of stroke, while intracerebral hemorrhagic for $10 \%$ and subarachnoid hemorrhagic constitute the remainder 3\% (Rink and Khanna, 2011).

The tissue injury of an organ also occurs at the time of its reoxygenation during the reperfusion. This may still be a more damaging process than ischemia itself. It is called reperfusion injury or ischemia-reperfusion injury ( $/ \mathrm{R})$. During the reperfusion, the restoration of blood flow worsens the tissue lesion and causes intense inflammatory response represented by the increase of reactive oxygen species (ROS), vascular endothelial lesions, increased vascular permeability and activation of platelets, neutrophils and cytokines (Stegner et al., 2019; Yang et al., 2019). Also, after stroke, cerebral edema occurs, which is one of the most relevant and responsible causes of neuronal death, as well as the development of brain lesions (Shamsaei et al., 2015; Piccardi et al., 2019). Thus, the pathology of cerebral I/R injury involves intense immune response, inflammation and cell death (Kriz et al., 2006; Piccardi et al., 2019).

In most western countries, alcohol is consumed as part of social gatherings, weddings and religious ceremonies and is enjoyed in moderation by the vast majority of the population. However, alcohol consumption also causes a wide range of problems. Alcohol abuse can lead to physical dependence associated with damage to target organs, particularly the brain and liver (Reilly and Noronha, 2014). Studies indicate that chronic low-level alcohol consumption protects the brain against $I / R$ injury. However, prolonged and excessive alcohol use has a detrimental effect on $\mathrm{I} / \mathrm{R}$ brain damage. This difference can be explained by a change in excitotoxicity of NMDA (Zhao et al., 2011; Carvalho et al., 2016). 
Several studies have shown that microRNAs (miRNAs), small non-coding RNAs with the role of transcriptional modulators of gene expression, plays a role in a large number of biological processes such as regulation of growth and differentiation cell proliferation, apoptosis, neuronal differentiation, neurogenesis, synaptic function and possibly in several neuronal disorders (Croce and Calin, 2005; Kosik and Krichevsky, 2005; Jeyaseelan et al., 2008; Dharap et al., 2009), including alcoholism (Miranda et al., 2010) and cerebral ischemia (Jeyaseelan et al., 2008; Dharap et al., 2009; Tan et al., 2009; Liu et al., 2010).

The role of microRNAs has been highlighted in the literature as biomarkers in various types of diseases, including neurological disorders. Future studies will show whether the modulation of specific miRNAs may be a therapeutic option to prevent pathophysiological events after ischemic and/or promoting cell regeneration and plasticity in nervous tissue. Accordingly, the identification of molecular markers in the serum involved in the pathogenesis of cerebral ischemia as well as alcoholism could help in the diagnosis and in the development of future therapeutic targets for these diseases (Silva et al., 2019).

In this context, there is limited information about the expression of miRNAs related to the mechanism of apoptosis in experimental brain ischemia and alcoholism. Along this line, we analyzed the serum expression of miRNAs-16 and 15b involved in apoptosis and miRNAs -21, -221 and -222 involved in anti-apoptotic process after induction of experimental transient focal cerebral ischemia and reperfusion, associated or not with chronic alcoholism in rats.

\section{MATERIAL AND METHODS}

This study was approved by the Ethics Committee on the Use of Animals of the Ribeirão Preto Medical School (CEUA-FMRP, Protocol n ${ }^{\circ}$ 279/200) and the experiments were carried out according to the Ethical Principles and Guidelines for Experiments on Animals. Fifty-six adult male rats (Rattus norvegicus) weighing 280-310 g were used. The animals were randomly divided into seven experimental groups: Control (C), 8 animals euthanized without being submitted to the surgical procedure; Sham (S), 8 control animals submitted to complete simulation of the surgical procedure but without obstruction of the middle cerebral artery (MCA) and then were euthanized; Ischemic (I), 8 animals submitted to focal ischemia by MCA occlusion (MCAO) for $90 \mathrm{~min}$ and then euthanized; Alcoholic (A), 8 animals received $20 \%$ ethanol diluted in water during 4 weeks and then were euthanized; Ischemic and Alcoholic $(\mathrm{I}+\mathrm{A}), 8$ animals received the same treatment from group A and after the period of four weeks were submitted to focal ischemia by MCAO during $90 \mathrm{~min}$, and then were euthanized; Ischemia-reperfusion (IR), 8 animals submitted to focal ischemia by MCAO during $90 \mathrm{~min}$, followed by reperfusion of $48 \mathrm{~h}$, and then were euthanized; Ischemia-reperfusion and Alcoholic $(\mathrm{IR}+\mathrm{A})$ : 8 animals received the same treatment from group A and, after the period of 4 weeks, were submitted to focal ischemia by MCAO during $90 \mathrm{~min}$, followed by reperfusion of $48 \mathrm{~h}$, and then were euthanized.

For animals of groups A, I+A and IR+A, we used the model of "semi-voluntary alcoholism" in which ethanol solution at $20 \%$ was the only liquid available to these animals. These experimental groups were conditioned to a brief period of adaptive phase consisted of 
the supply of ethanol in increasing weekly concentrations of 5, 10 and $20 \%$, starting the experimental phase after the third week of treatment.

\section{Induction of Cerebral Ischemia}

All animals were lightly anesthetized by halothane inhalation and intubated with an orotracheal cannula. During the ischemic period, arterial blood samples were collected in an amount of $0.5 \mathrm{~mL}$ twice through the caudal artery to determine blood glucose, $\mathrm{paCO}$, $\mathrm{paO} 2$ and $\mathrm{pH}$. MCAO was carried out by means of the retrograde introduction of a $2.5 \mathrm{~cm}$ long obstructive 4-0 mononylon suture with one end thickened with silicone over an extension of $5 \mathrm{~mm}$ into the external carotid artery. The suture was introduced until reached the common carotid artery and then cranially progressed through the internal carotid artery to obstruct the MCA.

The obstructive wire was removed after the period of ischemia; it was replaced with a temporary staple in the common and internal carotid arteries to prevent blood reflux. The proximal stump of the external carotid artery was connected, the temporary clamps were removed, and the tissues were sutured. The animals were sacrificed after the experimental procedure and the brain was removed for analysis of the ischemic area and histopathological analysis (Carlotti et al., 2001).

\section{Histopathological analysis}

The samples from groups I, IR, I+A and IR +A were included in paraffin and coronal sections $(2 \mathrm{~mm}$ ) were stained by Luxol Fast Blue to emphasize the cellular elements (neurons) which are stained pink-violet and myelin blue-green, on the same slide. Changes were assessed histopathological (neuronal injury, neuronal necrosis, edema and inflammatory infiltrate) in predetermined areas (area 1 - dorsolateral cortex, area 2 - 3 area and lateral cortex - striatum) using the left cerebral hemisphere (Figure 1).
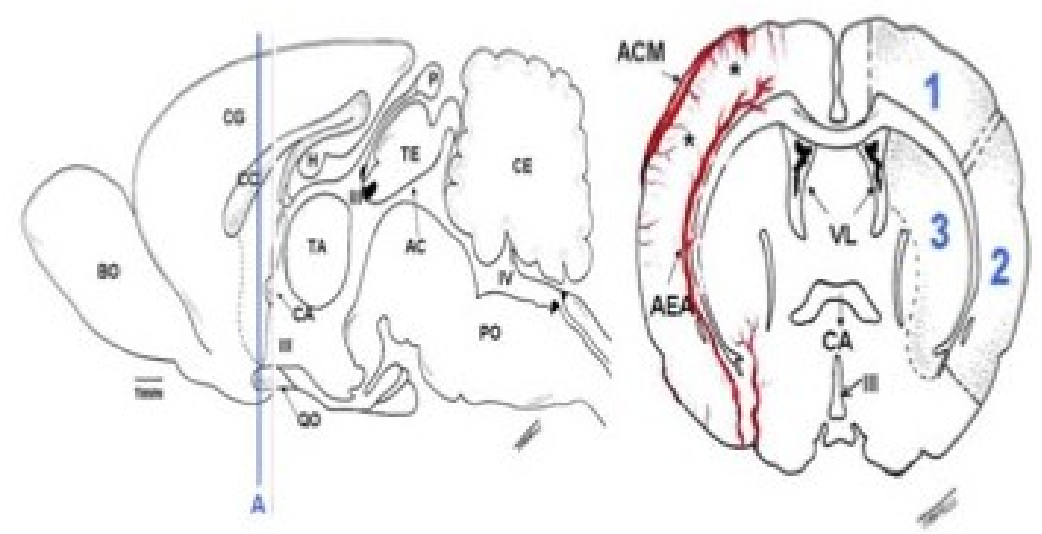

Figure 1. Median sagittal section with a view of the major structures observed in the adult rat brain. Line A - anterior or rostral (in blue) indicates the level of the coronal cut (on the right) and used for evaluation by ML, MET and IH. Brain aqueduct (AC); Middle cerebral artery (ACM); Perforating or central subcortical arteries (*); Anterior striated artery (AEA) - ACM branch; Olfactory bulb (BO); Anterior commissure (CA); Corpus callosum (CC); Cerebellum (CE); Cingulate (CG); Hippocampus (H); Pineal gland (P); Bridge (PO); Optic chiasm (QO); Thalamus (TA); Mesencephalic ceiling (TE); Third ventricle (III); Fourth ventricle (IV); Lateral ventricle (VL). The three areas evaluated in this study: dorsolateral cortex (1); lateral cortex (2), and striatum (3) (Tirapelli, 2007 - with permission). 


\section{Analysis of gene expression of serum miRNAs}

For the analysis of gene expression, $1 \mathrm{~mL}$ of blood was collected at different moments in each group. In groups $\mathrm{C}, \mathrm{S}$ and $\mathrm{A}$, blood samples were collected in anesthetized animals from the ventral tail artery after its cannulation. In groups I and I+A, the collection was performed immediately after the ischemic period $(90 \mathrm{~min})$ and in groups IR and IR+ A the collection was done after $48 \mathrm{~h}$ of reperfusion.

\section{RNA extraction and cDNA synthesis}

Blood samples were collected in EDTA-containing tube and centrifuged 10 min over $2500 \mathrm{rpm}$ and the "buffy coat" was transferred to a $15 \mathrm{~mL}$ tube. They were washed with buffer to lyse red blood cells and maintained for $15 \mathrm{~min}$ on ice. Further, followed by further centrifugation and further washed with a red blood cell lysis buffer. To obtain a pellet, the sample was centrifuged for $10 \mathrm{~min}$ at $2500 \mathrm{rpm}$. Added to $250 \mathrm{~mL}$ of PBS (phosphate-buffered saline) and $750 \mathrm{~mL}$ of Trizol (Invitrogen, USA) for freezing at $-80^{\circ} \mathrm{C}$. To check the integrity of the RNA obtained, at the end of stage described above, each sample was subjected to electrophoresis on agarose gel $1 \%$ to RNA. Complementary DNA (cDNA) was constructed by reverse transcription using the enzyme Superscript II and the DNA obtained was amplified by real-time quantitative PCR (RQ-PCR).

\section{qPCR microRNA}

The cDNA was perfomed using 5 ng of RNA and with the specific looped RT primer for the miR-219, miR-181b and miR-195 provided in the assay and reagents from the High Capacity C-DNA archive Kit (Applied Biosystems, Foster City, CA, USA) and 3.8U RNase Inhibitor (Applied Biosystems, Foster City, CA, USA) and incubated for $30 \mathrm{~min}$ at $16^{\circ} \mathrm{C}, 30 \mathrm{~min}$ at $42^{\circ} \mathrm{C}, 5$ min at $85^{\circ} \mathrm{C}$, and then held at $4^{\circ} \mathrm{C}$. Real-time PCR analysis of cDNA was performed at $95^{\circ} \mathrm{C}$ for $10 \mathrm{~min}$, followed by 40 cycles at $95^{\circ} \mathrm{C}$ for $15 \mathrm{~s}$ and $60^{\circ} \mathrm{C}$ for $1 \mathrm{~min}$ in the sequence detection system of an Abi Prism 7500 (Applied Biosystems, Foster City, CA, USA) in accordance with the manufacture's instructions using TaqMan ${ }^{\circledR}$ Reaction Master Mix (Applied Biosystems, USA) and the following primer: rno-miR-15b, rno-miR-16, rno-miR-21, rno-miR221 and rno-miR-222. The U6 (000391) gene was used as an endogenous control (housekeeping) for reaction of the microRNA. The data were analyzed (for microRNA) using the ABI-7500 SDS software and the variation of expression among samples was calculated by the $2^{-\Delta \Delta \mathrm{Ct}}$ method.

\section{Statistical Analysis}

Data were analyzed using one-way ANOVA followed by the Bonferroni post-test. GraphPad Prism software version 4.00 for Windows (GraphPad Software, San Diego, CA, USA) was used for analysis and the level of significance was set at $\mathrm{P}<0.05$ for two-tailed tests.

\section{RESULTS}

\section{Histopathologic Analysis}

Histopathological changes in the predetermined areas of the brain (area 1 - dorsal cortex, 2 - and 3 dorsolateral cortex - striatum) were observed in the groups submitted to 
MCAO (I, IR, I+A and IR+A). Figure 2 demonstrates the presence of neuronal injury, neuronal necrosis, edema and inflammatory infiltrate in these areas.

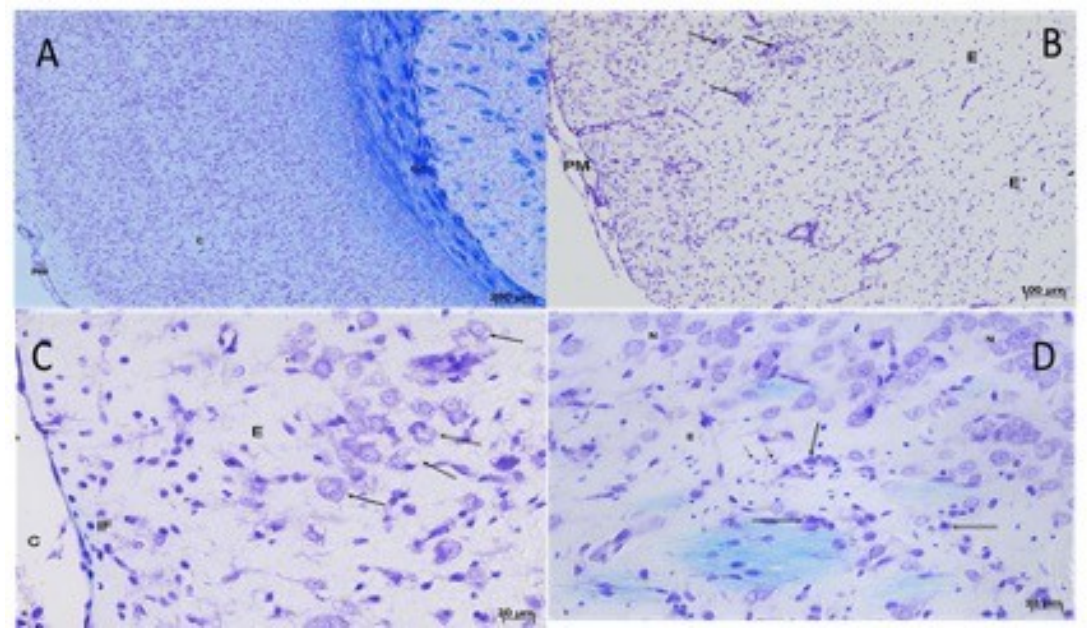

Figure 2. Photomicrographs, Luxol Fast Blue. A: Dorsolateral region of the frontal cortex of the $\mathrm{C}$ group; Cortex (C); corpus callosum (CC); (PM) 50x. B: Dorsolateral region of the frontal cerebral cortex of the I + A group. Note interstitial edema (E) with pyknotic nuclei and regions of inflammatory infiltration $(\rightarrow)$ along layer III capillaries; 100x. C: Dorsolateral region of the frontal cerebral cortex of group I. Note to the left, small inflammatory infiltrate (IF) from a capillary (C), in addition to interstitial edema (E) and several neurons with edemaciate cytoplasm and foamed nucleus $(\rightarrow)$; 400x. D: Medial region of the striatum of an animal of group I + A, showing the normal neurons $(\mathrm{N})$ near the side wall of the lateral ventricle (part not irrigated by the $\mathrm{ACM}$ ) and more laterally, region of interstitial edema (E ) with the presence of neurons with pyknotic nuclei (long arrows) and some neutrophils $(\rightarrow) ; 400 \mathrm{x}$.

\section{Analysis of gene expression of apoptotic serum miRNAs}

The serum expression of miRNA-15b (Figure 3 ) was similar in all groups $(\mathrm{P}=$ 0.9763). The serum expression of miRNA-16 was higher in the IR group compared to $\mathrm{C}$ and $\mathrm{S}$ (Figure 4, $\mathrm{P}=0.0119$ ).

\section{miR.15b}

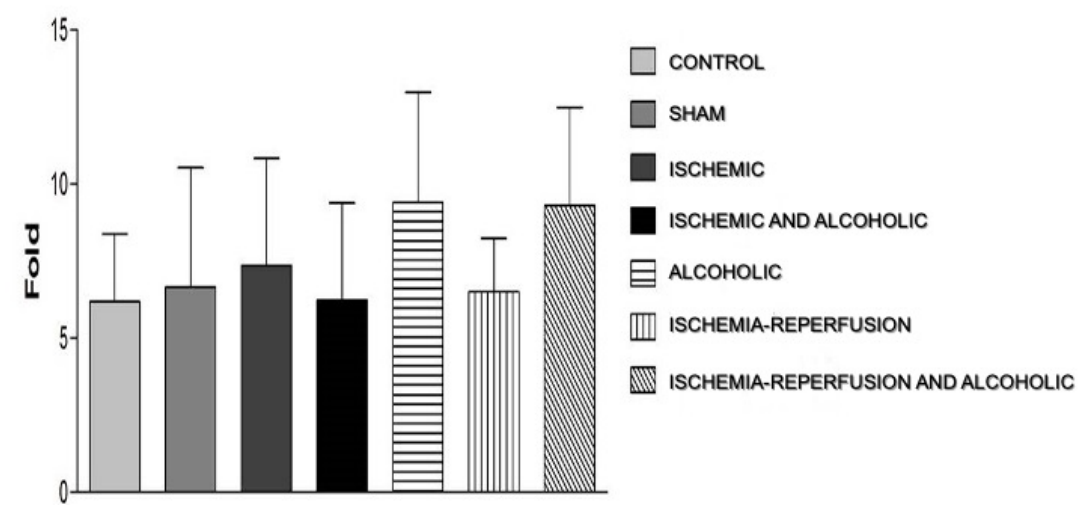

Figure 3. Representation of the mean ( \pm standard deviation) of gene expression of miR-15b serum between the rat treatment groups $(\mathrm{p}=0.9763$, One-Way ANOVA). 


\section{miR-16}

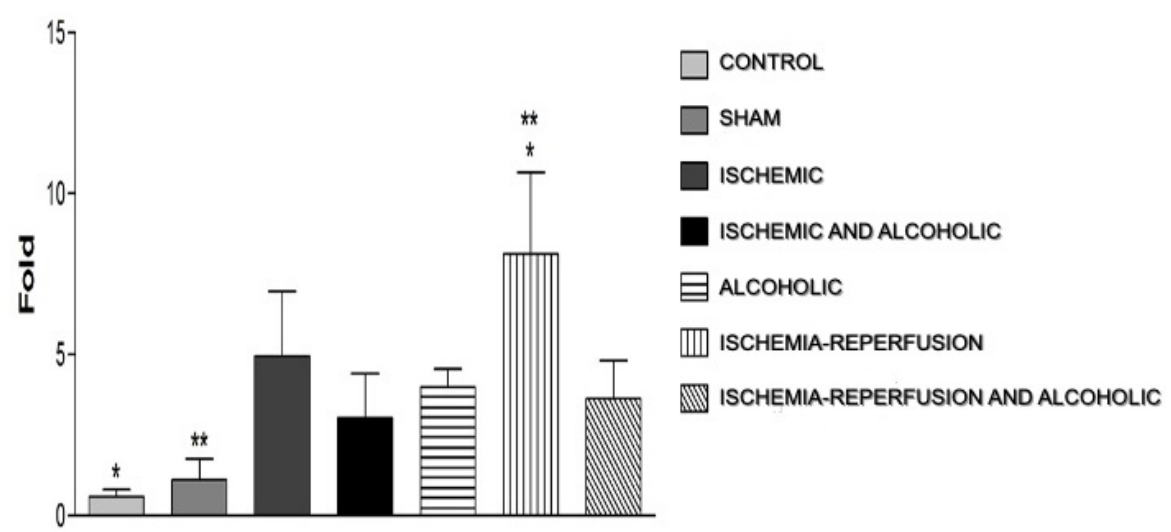

Figure 4. Representation of the mean ( \pm standard deviation) of gene expression of miR-16 serum between the rat treatment groups ( $\mathrm{p}=0.0119$, one-way ANOVA, * CxIR SxIR and $* * \mathrm{p}<0.05$, Bonferroni post-test).

\section{Analysis of gene expression of anti-apoptotic serum miRNAs}

The serum expression of miRNA-21 (Figure 5), miRNA-221 (Figure 6) and miRNA-222 (Figure 7) were low in all groups and no significant difference were observed among the groups (miRNA-21: $\mathrm{P}=0.8954$; miRNA-221: $\mathrm{P}=0.9934$ ). Although the expression of miRNA-222 seemed to be slightly increased in group A, it did not reach statistically significant difference $(\mathrm{P}=0.4473)$.

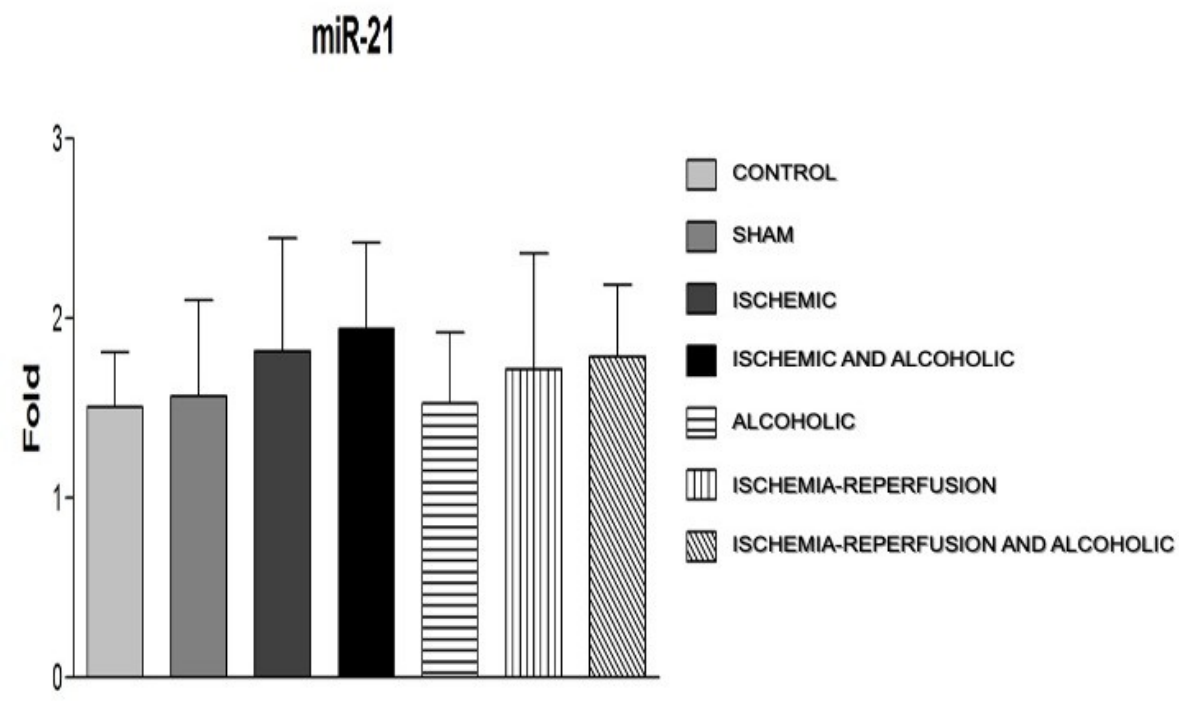

Figure 5. Representation of the mean ( \pm standard deviation) of gene expression of miR-21 serum between the rat treatment groups $(\mathrm{p}=0.8954$, One-Way ANOVA). 


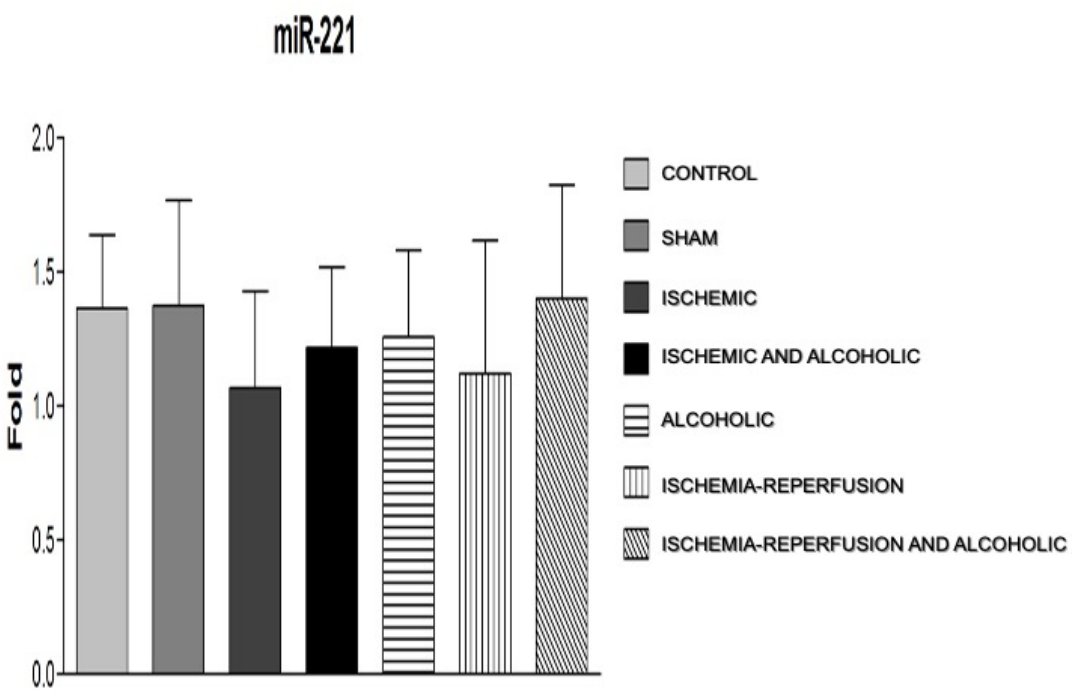

Figure 6. Representation of the mean ( \pm standard deviation) of gene expression of miR-221 serum between the rat treatment groups $(\mathrm{p}=0.9934$, One-Way ANOVA).

\section{miR.222}

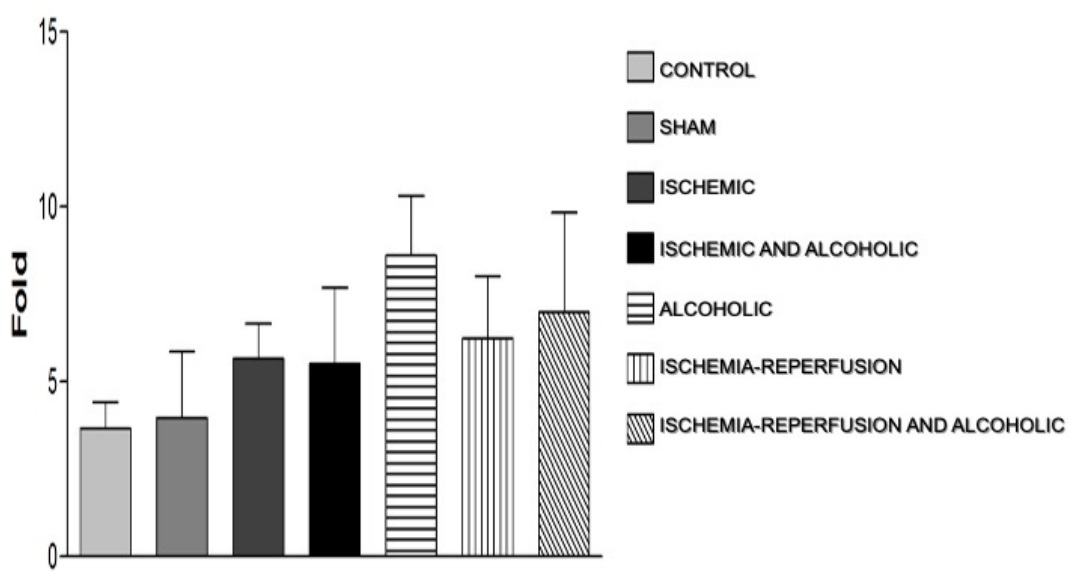

Figure 7. Representation of the mean ( \pm standard deviation) of gene expression of miR-222 serum between the rat treatment groups $(\mathrm{p}=0.4473$, One-Way ANOVA).

\section{DISCUSSION}

We examined the expression of serum miRNAs related to the mechanism of apoptosis (miRNA-15b and -16 - apoptotic and miRNA-21, -221 and -222 - anti-apoptotic) after focal cerebral ischemia for $90 \mathrm{~min}$ and reperfusion for 48 hours associated or not with alcoholism. We observed a significant increase in the serum expression of miRNA-16 in the IR group animals compared to $\mathrm{C}$ and $\mathrm{S}$ groups. 
Slota et al. (2019) reported that the central nervous system can respond to threats by inducing an inflammatory response, where it is tightly controlled. However, uncontrolled neuroinflammation is a hallmark of many neurological disorders. It also states that microRNAs are small, non-coding RNA molecules that are important for regulating many cellular processes. The ability of microRNAs to modulate inflammatory signaling is a promising research field, which has gained a lot of attention in recent years, including miR155, miR-146a, miR-124, miR-21 and let-7.

Studies involving the correlation between miRNAs and cerebral ischemia have been increasing, which demonstrate the great importance of this association (Jeyaseelan et al., 2008; Dharap et al., 2009; Tan et al., 2009; Liu et al., 2010). Jeyaseelan et al. (2008) demonstrated that the expression of various miRNAs was altered after $24 \mathrm{~h}$ and $48 \mathrm{~h}$ of reperfusion injury in brains of adult rats subjected to transient focal ischemia by means of MCAO. Furthermore, the impact of age and sex on miRNA expression after ischemic stroke in animal model had been investigated. An increase in the expression of miR-15a, miR-19b, miR-32 miR-136 and miR-199a-3p was demonstrated exclusively in adult females when compared to middle-aged females, males and middle-aged males. In general, the expression pattern of circulating miRNA suggests an early influence of age on stroke pathology, and, in later stage, the onset of sex as a factor for stroke severity (Selvamani et al., 2014).

Using blood samples obtained from young patients (18-49 years) with ischemic stroke, Tan et al. (2009) showed that, in addition to the disease progression, the subtype of stroke can also be identified by the miRNA profiling. The biological processes that are targets or are affected by differentially expressed miRNAs in stroke were analyzed and it was observed that miRNAs involved in the endothelial/vascular function, vascular remodeling, angiogenesis, hematopoeisis, immune response, hypoxic conditions and neural function showed differential expression profile as compared to the normal control. In addition, several miRNAs instead of a single miRNA showed changes during the progression of a disease and the dysregulated miRNAs have been detectable even after several months from the onset of stroke (Tan et al., 2009).

Another study analyzed and described the expression profile of the PARP and AIF proteins and miRNA-9 in rats submitted to focal cerebral ischemia, associated with a chronic alcoholism model using 20 adult Wistar rats subdivided into: control; ischemic; alcoholic and ischemic/ alcoholic for immunohistochemical analysis and expression of the miRNA-9. The authors found a reduction in PARP-1 protein expression and a positive AIF marking in the ischemic/alcoholic group; miRNA-9 was not significantly expressed (Abrahão et al., 2020).

Another study in patients was conducted to correlate whether concentrations of circulating miRNAs is associated with clinical outcomes beyond the stratification of some possible subtypes of cerebral ischemia. Plasma samples from patients with cerebral ischemia were screened by microarray to find differentially expressed miRNAs. The microarray revealed 12 differentially expressed miRNAs and miR-16 was the most significantly different. Bioinformatics analysis showed that potential miR-16 target genes were involved primarily in programmed cell death and in p53 signaling pathways. The elevated expression of miR-16 was associated with subtype of stroke involving total anterior circulation infarction and partial anterior circulation infarction, and with poor prognosis. This study indicated that a diagnostic method based on the rapid measurement of miR-16 in plasma has the potential to identify hyperacute cerebral infarction with high 
sensitivity and specificity, which would inform and improve early treatment and management decisions (Tian et al., 2016)

Accordingly, our study demonstrated that the serum expression of miRNA-16 was higher in IR group, and to a lesser extent in group I, when compared to $\mathrm{C}$ and $\mathrm{S}$ groups. These results were also similar to those observed by Tan et al. (2009) and Jeyaseelan et al. (2008) in which overexpression of miRNA-16 was observed in the serum and neural tissue of stroke patients and rats $24 \mathrm{~h}$ after MCAO reperfusion. Thus, we noted a higher correlation between serum expression of miRNA-16 in ischemic animals $48 \mathrm{~h}$ after reperfusion when compared to the acute ischemic animals, demonstrating that this miRNA is a potential serum biomarker for the study of apoptosis in cerebral ischemia, targeting the BCL-2 protein (anti-apoptotic). No association was observed when comparing the miRNA16 in chronic alcoholism.

Groups of miRNA-15a/16-1 and miRNA-15b/16-2 also regulate cell cycle and apoptosis by the fragmentation of cyclin D3 (CCND3), Cyclin E1 (CCNE1), CDK6 (Linsley et al., 2007; Liu et al., 2008), and anti-apoptotic gene BCL-2 in cells of many types of cancer (Aqeilan et al., 2009; Cimmino et al., 2005; Xia et al., 2008). In addition, some research related to miRNA-15a has demonstrated its contribution to the pathogenesis of ischemic vascular injury by means of the direct inhibition of the anti-apoptotic gene BCL-2. Their different expressions caused a significant reduction or increase in vascular endothelial cell death (Rink and Khanna, 2011).

We did not observe a significant difference in the expression of miRNA- $15 b$. Despite the knowledge about the relationship of the miRNA-15b with the cell cycle and apoptotic mechanisms in other tissues and diseases, no studies have demonstrated the association of this miRNA with experimental cerebral ischemia. Likewise, there was no evidence for an association with alcoholism and focal cerebral ischemia

Several reports have demonstrated the role of specific miRNAs in neuronal differentiation, neurogenesis, and neurological development (Kosik and Krichevsky, 2005). One of these reports showed that the absence of apoptosis is directly related to a specific miRNA, the miRNA-21. The blockade of aberrant expression of miRNA and gene expression products promotes normal differentiation of glial cells or induces apoptosis and maintains the cells in a continuous proliferative state. Thus, miRNA-21 was classified as an anti-apoptotic factor. The inhibition of its expression induces activation of caspases cascade and cell death by apoptosis. This suggests that overexpression miRNA-21 can act as a micro-oncogene, acting by blocking the gene expression of caspase cascade activators (Chan et al., 2005)

It was observed that the expression of miR-21 and miR-210 was dysregulated $24 \mathrm{~h}$ after ischemic brain injury and in neurons and astrocytes exposed to oxygen and glucose deprivation conditions (Jeyaseelan et al., 2008; Ziu et al., 2011). Similarly, another study reported overexpression of miR-21 and miR30b, 24 hours after traumatic brain injury in rats (Redell et al., 2009). In addition, Sathyan et al. (2007) showed that high doses (320mg/dl) ethanol suppresses the expression of specific miRNAs during cortical neurogenesis, mainly miRNA-21 and miRNA-335. Nevertheless, the mechanisms involved in ethanol-sensitive miRNAs regulation are divergent, since a GABA(A) receptor-dependent mechanism mediated miR-21, but not miR-335 suppression. Indeed, miRNAs sensitive to ethanol behave as agonists or antagonists for the survival and growth of the neuroepithelium and the removal coordinated miRNA-21 and miRNA-335 explains why ethanol leads to the death 
rather than proliferation of precursors of the cerebral cortex fetus. These data indicate that ethanol-sensitive miRNAs control important genes during development.

Our results showed a low gene expression of miRNA-21 in the blood of animals with no significant difference among the groups. However, in the literature, there is evidence that ethanol suppresses (Sathyan et al., 2007) or did not change miRNA-21 brain or serum expression in rodents (Ureña-Peralta et al., 2018; Silva et al., 2019). In our study, it is likely that the low concentration of ethanol used (20\%) was insufficient to induce suppressor function on the miRNA-21.

In gastric cancer, miRNAs-221 and -222 has found to target the protein PTEN, inhibiting proliferation and cell invasion, inducing cell apoptosis and enhancing the rate of sensitivity in the cells with high expression of PTEN in cells of this tumor (Chun-Zhi et al., 2010). Our findings demonstrated that the serum expression of miRNA-221 was low in all experimental groups and no association of its anti-apoptotic function was found with ischemia or chronic alcoholism. Regarding miRNA-222, we observed a slightly higher expression in groups $\mathrm{A}$ and $\mathrm{IR}+\mathrm{A}$ compared to groups $\mathrm{C}$ and $\mathrm{S}$, but it was not statistically significant. These results could indicate its anti-apoptotic effect, even in small degree, during $\mathrm{I} / \mathrm{R}$, mainly when associated with chronic alcoholism.

Although our results have not demonstrated a correlation of specific miRNAs studied in blood during brain ischemia and/or chronic alcoholism, we observed high expression of miRNA-16 in ischemia with reperfusion of $48 \mathrm{~h}$ when compared to control animals and sham. It is likely that specific signatures of miRNAs in blood are obtained from whole blood and can be used for the identification of biomarkers for diagnosis, prognosis or etiology of the disease.

\section{CONCLUSIONS}

The serum expression of miRNA-15b (apoptosis) and miRNAs -21, -221 and -222 were not affected by $\mathrm{I} / \mathrm{R}$ and ethanol, suggesting that there is no correlation between these miRNAs and the ischemia with or without reperfusion and/or chronic alcoholism. The overexpression of miRNA-16 (apoptosis) in the blood of animals in groups I and IR compared to groups $\mathrm{C}$ and $\mathrm{S}$ indicates that there is a good correlation with the ischemic process, mainly after reperfusion for 48 hours, but not associated with alcoholism.

\section{CONFLICTS OF INTEREST}

The authors declare no conflict of interest.

\section{REFERENCES}

\footnotetext{
Abrahão DPS, Neto FSL, Rodrigues AR, Cirino MLA, et al. (2020). AIF, PARP-1 AND MicroRNA-9 expression in cerebral ischemia associated to alcoholism experimental model. Int. J. Morphol. 38(3): 616-621.

Aqeilan RI, Calin GA and Croce CM (2009). MiR-15b and miR-16-1 in cancer: discovery, function and future perspectives. Cell Death Differ. 17(2): 215-220.

Carlotti CG, Colli BO and Kazuo JY (2001). Evaluation of brain ischemia by mitochondrial respiration: experimental model. Arq. Neuropsiquiatr. 59(2-B): 365-371.

Carvalho CA, Tirapelli DP, Rodrigues AR, Lizarte FS Neto, et al. (2016). Morphological and immunohistochemical analysis of apoptosis in the cerebellum of rats subjected to focal cerebral ischemia with or without alcoholism model. Acta Cir. Bras. 31(9): 629-637.
} 
Chan JA, Krichevsky AN and Kosik KS (2005). MicroRNA-21 is an antiapoptotic factor in human glioblastoma cells. Cancer Res. 65(14): 6029-6033.

Chun-zhi Z, Lei H, An-ling Z, Yan-chao F, et al. (2010). MicroRNA-221 and microRNA-222 regulate gastric carcinoma cell proliferation and radioresistance by targeting PTEN. BMC Cancer. 10: 367-377.

Cimmino A, Calin GA, Fabbri M, Iorio MV, et al. (2005). MiR-15 and miR-16 induce apoptosis by targeting BCL2. Proc. Natl. Acad. Sci. USA. 102: 13944-13949.

Croce CM and Calin GA (2005). miRNAs, cancer, and stem cell division. Cell. 122(1): 6-7.

Dharap A, Bowen K, Place R, LI, LC and R (2009). Transient focal ischemia induces extensive temporal changes in rat cerebral microRNAome. J. Cereb. Blood Flow Metab. 29(4): 675-687.

Jeyaseelan K, Lim KY and Armugam A (2008). MicroRNA Expression in the Blood and Brain of Rats Subjected to Transient Focal Ischemia by Middle Cerebral Artery Occlusion. Stroke. 39: 959-966.

Kosik KS and Krichevsky AM (2005). The elegance of the microRNAs: a neuronal perspective. Neuron. 47: 779-782.

Kriz J (2006). Inflammation in ischemia brain injury: timing is important. Crit. Rev. Neurocrit. Care. 18: 145-157.

Linsley PS, Schelter J, Burchard J, Kibukawa M, et al. (2007). Transcripts targeted by the microRNA-16 family cooperatively regulate cell cycle progression. Mol. Cell Biol. 27: 2240-2252.

Liu DZ, Tian Y, Ander BP, Xu H, et al. (2010). Brain and blood microRNA expression profiling of ischemic stroke, intracerebral hemorrhage, and kainate seizures. Journal of Cerebral Blood Flow \& Metabolism. 30: 92-101.

Qin L, Hanjiang F, Fang S, Haoming Z, et al. (2008). MiR-16 family induces cell cycle arrest by regulating multiple cell cycle genes. Nucleic Acids Res. 36: 5391-5404.

Miranda RC, Pietrzykowski AZ, Tang Y and Sathyan P (2010). MicroRNAs: master regulators of ethanol abuse and toxicity? Alcohol Clin. Exp. Res. 34 (4): 575-587.

Piccardi B, Biagini S, Iovene V and Palumbo V (2019). Blood Biomarkers of Parenchymal Damage in Ischemic Stroke Patients Treated With Revascularization Therapies. Biomark Insights. 24 14: 19.

Redell JB, Liu Y and Dash PK (2009). Traumatic brain injury alters expression of hippocampal microRNAs: potential regulators of multiple pathophysiological processes. J. Neurosci. Res. 87: 1435-1448.

Reilly MT and Noronha AK (2014). Perspectives on the neuroscience of alcohol from the National Institute on Alcohol Abuse and Alcoholism. Handb. Clin. Neurol. 125: 15-29.

Rink C and Khanna S (2011). MicroRNA in ischemic stroke etiology and pathology. Physiological Genomics. 43(10): 521-528.

Sathyan P, Golden HB and Miranda RC (2007). Competing interactions between micro-RNAs determine neural progenitor survival and proliferation after ethanol exposure: evidence from an ex vivo model of the fetal cerebral cortical neuroepithelium. J. Neurosci. 27(32): 8546-8557.

Selvamani A, Williams MH, Miranda RC and Sohrabji F (2014). Circulating miRNA profiles provide a biomarker for severity of stroke outcomes associated with age and sex in a rat model. Clin. Sci. (Lond). 12(2): 77-89.

Shamsaei N, Erfani E, Fereidoni M and Shahbazi A (2015). Exercise preconditioning exhibits neuroprotective effects on hippocampal CA1 neuronal damage after cerebral ischemia. Neural Regen. Res. 10(8): 1245-1250.

Silva JPD, Lizarte Neto FS, Cirino MLA, Carvalho CAM, et al. (2019). Analysis of Caspase-9 protein and microRNAs miR-21, miR-126 and miR-155 related to the apoptosis mechanism in the cerebellum of rats submitted to focal cerebral ischemia associated with an alcoholism model. Arq. Neuropsiquiatr. 77(10): 689-695.

Slota JA and Booth SA (2019). MicroRNAs in Neuroinflammation: Implications in Disease Pathogenesis, Biomarker Discovery and Therapeutic Applications. Noncoding RNA. 5(2): 35.

Stegner D, Klaus V and Nieswandt B (2019). Platelets as Modulators of Cerebral Ischemia/Reperfusion Injury. Front Immunol. 10: 2505.

Tan KS, Armugan A, Sepramaniam S, Lim KY, et al. (2009). Expression profile of microRNAs in young stroke patients. PloS One. 4(11): e7689.

Tian C, Li Z, Yang Z, Huang Q, et al. (2016). Plasma MicroRNA-16 Is a Biomarker for Diagnosis, Stratification, and Prognosis of Hyperacute Cerebral Infarction. PloS One. 11(11): e0166688.

Tirapelli DPC (2007). Expressão gênica da apoptose celular na isquemia cerebral focal em rato e a ação neuroprotetora da hipotermia e do cetoprofeno. Ph.D. thesis in Clinical Medicine, Ribeirão Preto Medical School. University of São Paulo, Ribeirão Preto, Brazil.

Xia L, Zhang D, Du R, Pan Y, et al. (2008). MiR-15b and miR-16 modulate multidrug resistance by targeting BCL2 in human gastric cancer cells. Int. J. Cancer. 123: 372-379.

Yang Q, Huang Q, Hu Z, Tang X. Potential Neuroprotective Treatment of Stroke: Targeting Excitotoxicity, Oxidative Stress, and Inflammation. Front Neurosci. 13: 1036

Zhao H, Mayhan WG, Arrick DM, Xiong W, et al. (2011). Dose-Related Influence of Chronic Alcohol Consumption on Cerebral Ischemia/Reperfusion Injury. Alcohol Clin. Exp. Res. 35(7): 1265-1269.

Ziu M, Fletcher L, Rana S, Jimenez DF, et al. (2011). Temporal differences in microRNA expression patterns in astrocytes and neurons after ischemic injury. PloS One. 6(2): e14724. 